\title{
The Erdős-Hajnal property for graphs with no fixed cycle as a pivot-minor
}

\author{
Jaehoon Kim* \\ Department of Mathematical Sciences \\ KAIST \\ Daejeon, Korea \\ jaehoon.kim@kaist.ac.kr
}

\author{
Sang-il Oum ${ }^{\dagger}$ \\ Discrete Mathematics Group \\ Institute for Basic Science (IBS) \\ Daejeon, Korea \\ Department of Mathematical Sciences \\ KAIST \\ Daejeon, Korea \\ sangil@ibs.re.kr
}

Submitted: Apr 23, 2020; Accepted: Mar 22, 2021; Published: Apr 9, 2021

(C) The authors. Released under the CC BY-ND license (International 4.0).

\begin{abstract}
We prove that for every integer $k$, there exists $\varepsilon>0$ such that for every n-vertex graph $G$ with no pivot-minor isomorphic to $C_{k}$, there exist disjoint sets $A, B \subseteq V(G)$ such that $|A|,|B| \geqslant \varepsilon n$, and $A$ is either complete or anticomplete to $B$. This proves the analog of the Erdős-Hajnal conjecture for the class of graphs with no pivot-minor isomorphic to $C_{k}$.
\end{abstract}

Mathematics Subject Classifications: 05CC55, 05C75

\section{Introduction}

In this paper all graphs are simple, having no loops and no parallel edges. For a graph $G$, let $\omega(G)$ be the maximum size of a clique, that is a set of pairwise adjacent vertices and let $\alpha(G)$ be the maximum size of an independent set, that is a set of pairwise non-adjacent vertices. Erdős and Hajnal [9] proposed the following conjecture in 1989.

Conjecture 1 (Erdös and Hajnal [9]). For every graph $H$, there is $\varepsilon>0$ such that all graphs $G$ with no induced subgraph isomorphic to $H$ satisfies

$$
\max (\alpha(G), \omega(G)) \geqslant|V(G)|^{\varepsilon} .
$$

\footnotetext{
* Supported by the POSCO Science Fellowship of POSCO TJ Park Foundation and by the KAIX Challenge program of KAIST Advanced Institute for Science-X.

†Supported by the Institute for Basic Science (IBS-R029-C1).
} 
This conjecture still remains open. See [5] for a survey on this conjecture. We can ask the same question for weaker containment relations. Recently Chudnovsky and Oum [6] proved that this conjecture holds if we replace "induced subgraphs" with "vertex-minors" as follows. This is weaker in the sense that every induced subgraph $G$ is a vertex-minor of $G$ but not every vertex-minor of $G$ is an induced subgraph of $G$.

Theorem 2 (Chudnovsky and Oum [6]). For every graph $H$, there exists $\varepsilon>0$ such that every graph with no vertex-minors isomorphic to $H$ satisfies

$$
\max (\alpha(G), \omega(G)) \geqslant|V(G)|^{\varepsilon} .
$$

We ask whether Conjecture 1 holds if we replace "induced subgraphs" with "pivotminors" as follows.

Conjecture 3. For every graph $H$, there exists $\varepsilon>0$ such that every graph $G$ with no pivot-minor isomorphic to $H$ satisfies

$$
\max (\alpha(G), \omega(G)) \geqslant|V(G)|^{\varepsilon} .
$$

The detailed definition of pivot-minors will be presented in Section 3. For now, note that the analog for vertex-minors is weakest, the analog for pivot-minors is weaker than that for induced subgraphs but stronger than that for vertex-minors. This is because every induced subgraph of $G$ is a pivot-minor of $G$, and every pivot-minor of $G$ is a vertex-minor of $G$. In other words, Conjecture 1 implies Conjecture 3 and Conjecture 3 implies Theorem 2. We verify Conjecture 3 for $H=C_{k}$, the cycle graph on $k$ vertices as follows.

Theorem 4. For every $k \geqslant 3$, there exists $\varepsilon>0$ such that every graph with no pivot-minor isomorphic to $C_{k}$ satisfies

$$
\max (\alpha(G), \omega(G)) \geqslant|V(G)|^{\varepsilon} .
$$

We actually prove a stronger property, as Chudnovsky and Oum [6] did. Before stating this property, let us first state a few terminologies. A class $\mathcal{G}$ of graphs closed under taking induced subgraphs is said to have the Erdös-Hajnal property if there exists $\varepsilon>0$ such that every graph $G$ in $\mathcal{G}$ satisfies

$$
\max (\alpha(G), \omega(G)) \geqslant|V(G)|^{\varepsilon} .
$$

A class $\mathcal{G}$ of graphs closed under taking induced subgraphs is said to have the strong Erdös-Hajnal property if there exists $\varepsilon>0$ such that every $n$-vertex graph in $\mathcal{G}$ with $n>1$ has disjoint sets $A, B$ of vertices such that $|A|,|B| \geqslant \varepsilon n$ and $A$ is either complete or anti-complete to $B$. It is an easy exercise to show that the strong Erdös-Hajnal property implies the Erdős-Hajnal property, see $[1,10]$.

Chudnovsky and Oum [6] proved that the class of graphs with no vertex-minors isomorphic to $H$ for a fixed graph $H$ has the strong Erdős-Hajnal property, implying Theorem 2. We propose its analog for pivot-minors as a conjecture, which implies the theorem 
of Chudnovsky and Oum [6]. Note that this conjecture is not true if we replace the pivotminor with induced graphs. For example, the class of triangle-free graphs does not have the strong Erdős-Hajnal property [10].

Conjecture 5. For every graph $H$, there exists $\varepsilon>0$ such that for all $n>1$, every $n$-vertex graph with no pivot-minor isomorphic to $H$ has two disjoint sets $A, B$ of vertices such that $|A|,|B| \geqslant \varepsilon n$ and $A$ is complete or anti-complete to $B$.

We prove that this conjecture holds if $H=C_{k}$. In other words, the class of graphs with no pivot-minor isomorphic to $C_{k}$ has the strong Erdös-Hajnal property as follows. This implies Theorem 4.

Theorem 6. For every integer $k \geqslant 3$, there exists $\varepsilon>0$ such that for all $n>1$, every $n$-vertex graph with no pivot-minor isomorphic to $C_{k}$ has two disjoint sets $A, B$ of vertices such that $|A|,|B| \geqslant \varepsilon n$ and $A$ is complete or anti-complete to $B$.

This paper is organized as follows. In Section 2, we will introduce basic definitions and review necessary theorems of Rödl [20] and Bonamy, Bousquet, and Thomassé [2]. In Section 3, we will present several tools to find a pivot-minor isomorphic to $C_{k}$. In particular, it proves that a long anti-hole contains $C_{k}$ as a pivot-minor. In Section 4, we will present the proof of the main theorem, Theorem 6. In Section 5, we will relate our theorem to the problem on $\chi$-boundedness, and discuss known results and open problems related to polynomial $\chi$-boundedness and the Erdős-Hajnal property.

\section{Preliminaries}

Let $\mathbb{N}$ be the set of positive integers and for each $n \in \mathbb{N}$, we write $[n]:=\{1,2, \ldots, n\}$. For a graph $G=(V, E)$, let $\bar{G}=\left(V,\left(\begin{array}{c}V \\ 2\end{array}\right)-E\right)$ be the complement of $G$. We write $\Delta(G)$ and $\delta(G)$ to denote the maximum degree of $G$ and the minimum degree of $G$ respectively.

Let $T$ be a tree rooted at a specified node $v_{r}$, called the root. If the path from $v_{r}$ to a node $y$ in $T$ contains $x \in V(T)-\{y\}$, we say that $x$ is an ancestor of $y$, and $y$ is a descendant of $x$. If one of $x$ and $y$ is an ancestor of the other, we say that $x, y$ are related. We say that two disjoint sets $X$ and $Y$ of nodes of $T$ are unrelated if no pairs of $x \in X$ and $y \in Y$ are related.

For disjoint vertex sets $X$ and $Y$, we say $X$ is complete to $Y$ if every vertex of $X$ is adjacent to all vertices of $Y$. We say $X$ is anti-complete to $Y$ if every vertex of $X$ is non-adjacent to $Y$. A pure pair of a graph $G$ is a pair $(A, B)$ of disjoint subsets of $V(G)$ such that $A$ is complete or anticomplete to $B$.

For a vertex $u$, let $N_{G}(u)$ denote the set of neighbors of $u$ in $G$. For each $U \subseteq V(G)$, we write

$$
N_{G}(U):=\bigcup_{u \in U} N_{G}(u)-U
$$

The following lemma is proved in Section 2 of [2]. 
Lemma 7 (Bonamy, Bousquet, and Thomassé [2]). For every connected graph G and a vertex $v_{r} \in V(G)$, there exist an induced subtree $T$ of $G$ rooted at $v_{r}$ and a function $r: V(G) \rightarrow V(T)$ satisfying the following.

(T1) $r\left(v_{r}\right)=v_{r}$ and for each $u \in V(G)-\left\{v_{r}\right\}$, the vertex $r(u)$ is a neighbor of $u$. In particular, $T$ is a dominating tree of $G$.

(T2) If $r(x)$ and $r(y)$ are not related, then $x y \notin E(G)$.

Rödl [20] proved the following theorem. Its weaker version was later proved by Fox and Sudakov [11] without using the regularity lemma. A set $U$ of vertices of $G$ is an $\varepsilon$-stable set of a graph $G$ if $G[U]$ has at most $\varepsilon\left(\begin{array}{c}|U| \\ 2\end{array}\right)$ edges. Similarly, $U$ is an $\varepsilon$-clique of a graph $G$ if $G[U]$ has at least $(1-\varepsilon)\left(\begin{array}{c}|U| \\ 2\end{array}\right)$ edges.

Theorem 8 (Rödl [20]). For all $\varepsilon>0$ and a graph $H$, there exists $\delta>0$ such that every $n$-vertex graph $G$ with no induced subgraph isomorphic to $H$ has an $\varepsilon$-stable set or an $\varepsilon$-clique of size at least $\delta n$.

We will use the following simple lemma. We present its proof for completeness.

Lemma 9. Let $G$ be a graph. Every $\varepsilon$-stable set $U$ of $G$ has a subset $U^{\prime}$ of size at least $|U| / 2$ with $\Delta\left(G\left[U^{\prime}\right]\right) \leqslant 4 \varepsilon\left|U^{\prime}\right|$.

Proof. Let $U^{\prime}$ be the set of vertices of degree at most $2 \varepsilon|U|$ in $G[U]$. Because

$$
\sum_{v \in U} \operatorname{deg}_{G[U]}(v)<\varepsilon|U|^{2}
$$

we have $\left|U^{\prime}\right| \geqslant|U| / 2$. Moreover, for each vertex $v \in U^{\prime}$, we have $\operatorname{deg}_{G\left[U^{\prime}\right]}(v) \leqslant 2 \varepsilon|U| \leqslant$ $4 \varepsilon\left|U^{\prime}\right|$.

Using Lemma 9, we can deduce the following corollary of Theorem 8.

Corollary 10. For all $\alpha>0$ and a graph $H$, there exists $\delta>0$ such that every graph $G$ with no induced subgraph isomorphic to $H$ has a set $U \subseteq V(G)$ with $|U| \geqslant \delta|V(G)|$ such that either $\Delta(G[U]) \leqslant \alpha|U|$ or $\Delta(\bar{G}[U]) \leqslant \alpha|U|$.

The following easy lemma will be used to find a connected induced subgraph inside the output of Corollary 10. We omit its easy proof.

Lemma 11. A graph $G$ has a pure pair $(A, B)$ such that $|A|,|B| \geqslant|V(G)| / 3$ or has a connected induced subgraph $H$ such that $|V(H)| \geqslant|V(G)| / 3$.

Lemma 12 (Bonamy, Bousquet, and Thomassé [2, Lemma 3]). Let $T$ be a tree rooted at $v_{r}$ and $w: V(T) \rightarrow \mathbb{R}$ be a non-negative weight function on $V(T)$ with $\sum_{x \in V(T)} w(x)=1$. Then there exists either a path $P$ from $v_{r}$ with weight at least $1 / 4$ or two unrelated sets $A$ and $B$ both with weight at least $1 / 4$. 


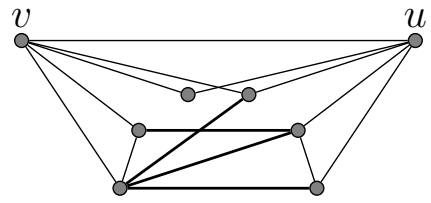

G

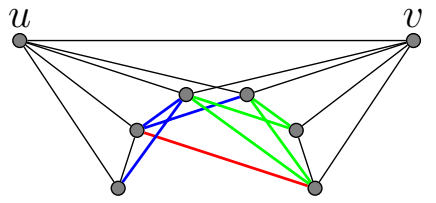

$G \wedge u v$

Figure 1: Pivoting uv.

A hole is an induced cycle of length at least 5 .

Lemma 13 (Bonamy, Bousquet, and Thomassé [2, Lemma 4]). For given $k \geqslant 3$, there exist $\alpha=\alpha(k)>0$ and $\varepsilon=\varepsilon(k)>0$ such that for any $n$-vertex graph $G$ with $n \geqslant 2$ and $\Delta(G) \leqslant \alpha n$, if $G$ has no holes of length at least $k$ and has a dominating induced path, then $G$ contains a pair $(A, B)$ of disjoint vertex sets such that $A$ is anticomplete to $B$ and $|A|,|B| \geqslant \varepsilon n$.

\section{Finding a cycle as a pivot-minor}

For a given graph $G$ and an edge $u v$, a graph $G \wedge u v$ obtained from $G$ by pivoting $u v$ is defined as follows. Let $V_{1}=N_{G}(u) \cap N_{G}(v), V_{2}=N_{G}(u)-N_{G}(v), V_{3}=N_{G}(v)-N_{G}(u)$. Then $G \wedge u v$ is the graph obtained from $G$ by complementing adjacency between vertices between $V_{i}$ and $V_{j}$ for all $1 \leqslant i<j \leqslant 3$ and swapping the label of $u$ and $v$. See Figure 1 for an illustration. We say that $H$ is a pivot-minor of $G$ if $H$ can be obtained from $G$ by deleting vertices and pivoting edges. For this paper, we will also say that $H$ is a pivot-minor of $G$, when $G$ has a pivot-minor isomorphic to $H$. A pivot-minor $H$ of $G$ is proper if $|V(H)|<|V(G)|$.

We describe several scenarios for constructing $C_{k}$ as a pivot-minor. The following proposition is an easy one; One can obtain a desired pivot-minor from a longer cycle of the same parity.

Proposition 14. For $m \geqslant k \geqslant 3$ with $m \equiv k(\bmod 2)$, the cycle $C_{m}$ has a pivot-minor isomorphic to $C_{k}$.

Proof. We proceed by induction on $m-k$. We may assume that $m>k$. Let $x y$ be an edge of $C_{m}$. Then $\left(C_{m} \wedge x y\right)-x-y$ is isomorphic to $C_{m-2}$, which contains a pivot-minor isomorphic to $C_{k}$ by the induction hypothesis.

Proposition 15. For integers $k \geqslant 3$ and $m \geqslant \frac{3}{2} k+6$, the graph $\overline{C_{m}}$ has a pivot-minor isomorphic to $C_{k}$.

Before proving Proposition 15, we present a simple lemma on partial complements of the cycle graph. The partial complement ${ }^{1} G \oplus S$ of a graph $G$ by a set $S$ of vertices is a

\footnotetext{
${ }^{1}$ We found this concept in a paper by Kamiński, Lozin, and Milanič [14], though it may have been studied previously, as it is a natural concept.
} 

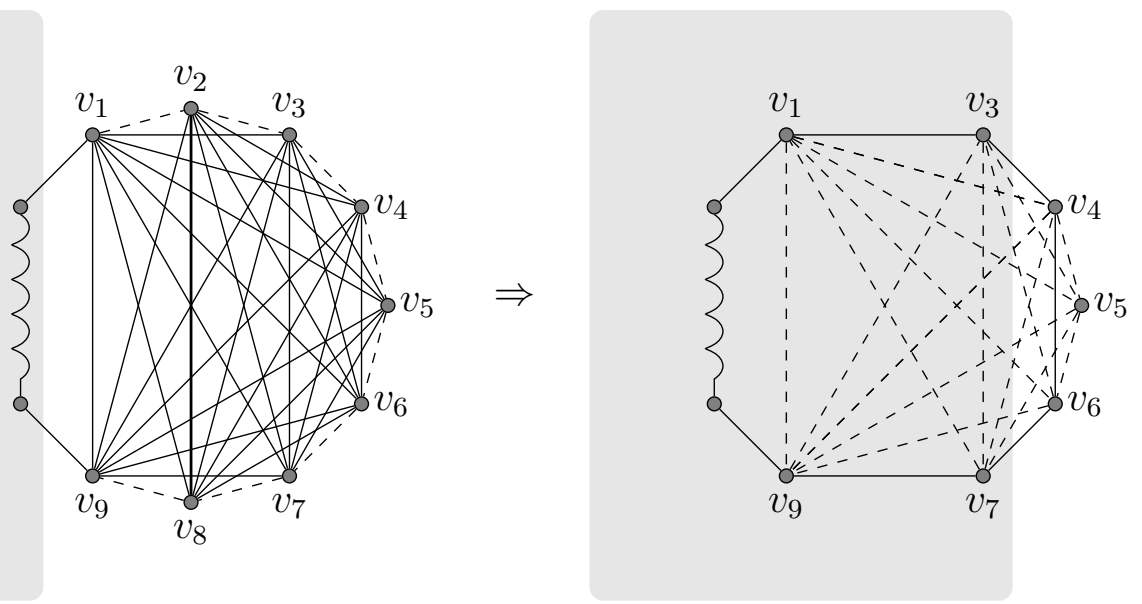

Figure 2: Obtaining an $(s-2,3)$-cycle from an $(s, 9)$-cycle when $s>9$ in the proof of Lemma 16.

graph obtained from $G$ by changing all edges within $S$ to non-edges and non-edges within $S$ to edges.

For $s \geqslant t \geqslant 0$, we say that $G$ is an $(s, t)$-cycle if $G$ is isomorphic to a graph $C_{s} \oplus X$ for a set $X$ of $t$ consecutive vertices in the cycle $C_{s}$.

Lemma 16. Let $s \geqslant t \geqslant 6$. An $(s, t)$-cycle contains a pivot-minor isomorphic to an $(s-2, t-6)$-cycle.

Proof. Let $v_{1}, \ldots, v_{s}$ be the vertices of $C_{s}$ in the cyclic order where $X=\left\{v_{1}, \ldots, v_{t}\right\}$. Then it is easy to check that $\left(C_{s} \oplus X\right) \wedge v_{2} v_{t-1}-\left\{v_{2}, v_{t-1}\right\}$ is isomorphic to $C_{s-2} \oplus X^{\prime}$ where $X$ consists of $t-6$ consecutive vertices on the cycle. See Figure 2 .

Proof of Proposition 15. As $\overline{C_{m}}$ is an $(m, m)$-cycle, by Lemma $16, \overline{C_{m}}$ contains a pivotminor isomorphic to an $(m-2 i, m-6 i)$-cycle for all $i \leqslant m / 6$.

Let us fix $i=\lceil(k-2) / 4\rceil$. Then $m-6 i \geqslant m-6 \cdot(k+1) / 4 \geqslant 9 / 2$ and therefore $\overline{C_{m}}$ contains a pivot-minor $H$ isomorphic to an $(m-2 i, m-6 i)$-cycle and $m-6 i \geqslant 5$. We may assume that $H=C_{m-2 i} \oplus X$ where $C_{m-2 i}=v_{1} \cdots v_{m-2 i}$ and $X=\left\{v_{4 i+1}, \ldots, v_{m-2 i}\right\}$.

Note that $H$ contains an induced cycle $C=v_{1} \cdots v_{4 i} v_{4 i+1} v_{m-2 i} v_{1}$ of length $4 i+2 \geqslant k$. If $k$ is even, then by Proposition 14, $H$ contains a pivot-minor isomorphic to $C_{k}$. So we may assume that $k$ is odd and therefore $|V(C)|=4 i+2 \geqslant k+1$.

Let $x=v_{m-2 i}, y=v_{4 i+1}$ be the two vertices in $V(C) \cap X$. Since $m-6 i \geqslant 5$, there is a common neighbor $z$ of $x$ and $y$ in $X$. Then $z$ has exactly two neighbors $x$ and $y$ in $V(C)$. Then $H[V(C) \cup\{z\}] \wedge y z-y-z$ is a cycle of length $4 i+1$. Since $4 i+1 \geqslant k$, by Proposition 14, it contains a pivot-minor isomorphic to $C_{k}$.

A generalized fan is a graph $G$ with a specified vertex $c$, called the center, such that $G-c$ is an induced path of length at least 1, called the main path of $G$ and both ends of the main path are adjacent to $c$. If $c$ is adjacent to all vertices of $G-c$, then $G$ is called a fan. 
An interval of a generalized fan with a center $c$ is a maximal subpath of the main path having no internal vertex adjacent to $c$. The length of an interval is its number of edges. A generalized fan is an $\left(a_{1}, \ldots, a_{s}\right)$-fan if the lengths of intervals are $a_{1}, \ldots, a_{s}$ in order. Note that an $\left(a_{1}, \ldots, a_{s}\right)$-fan is also an $\left(a_{s}, \ldots, a_{1}\right)$-fan. An $\left(a_{1}, \ldots, a_{s}\right)$-fan is a $k$-good fan if $a_{1} \geqslant k-2$ or $a_{s} \geqslant k-2$. An $\left(a_{1}, \ldots, a_{s}\right)$-fan is a strongly $k$-good fan if $s \geqslant 2$ and either $a_{1} \geqslant k-2$ and $a_{s}$ is odd, or $a_{s} \geqslant k-2$ and $a_{1}$ is odd. It is easy to observe that every $k$-good fan has a hole of length at least $k$. However, that does not necessarily lead to a pivot-minor isomorphic to $C_{k}$ because of the parity issues. In the next proposition, we show that every strongly $k$-good fan has a pivot-minor isomorphic to $C_{k}$.

Proposition 17. Let $k \geqslant 5$ be an integer. Every strongly $k$-good fan has a pivot-minor isomorphic to $C_{k}$.

Proof. Let $G$ be an $\left(a_{1}, \ldots, a_{s}\right)$-fan such that $s \geqslant 2, a_{1} \geqslant k-2$, and $a_{s}$ is odd. We proceed by the induction on $|V(G)|$. We may assume that $G$ has no proper pivot-minor that is a strongly $k$-good fan. Note that $C_{a_{1}+2}$ is an induced subgraph of $G$, hence if $a_{1} \equiv k$ $(\bmod 2)$, then $C_{k}$ is isomorphic to a pivot-minor of $G$ by Proposition 14. Thus we may assume that $a_{1} \not \equiv k(\bmod 2)$ and so $a_{1} \geqslant k-1$.

If $a_{i}$ is odd for some $1<i<s$, then $G$ contains a smaller strongly $k$-good fan by taking the first $i$ intervals, contradicting our assumption. Thus $a_{i}$ is even for all $1<i<s$. If $a_{i} \geqslant 3$ for some $i>1$, then let $u v$ be an internal edge of the $i$-th interval. Then $G \wedge u v-u-v$ is a strongly $k$-good fan, contradicting our assumption. Thus, we may assume that $a_{i} \leqslant 2$ for all $i>1$ and so $G$ is an $\left(a_{1}, 2, \ldots, 2,1\right)$-fan.

Let $x y$ be the last interval of $G$ with length 1 . Then $G \wedge x y-x-y$ is a $\left(a_{1}, 2, \ldots, 2,1\right)$ fan with $s-1$ intervals. By the assumption, we may assume that $s=2$ and $G \wedge x y-x-y$ is an $\left(a_{1}-1\right)$-fan with one interval, which is a cycle with $a_{1}+1$ edges. As $a_{1}+1 \geqslant k$ and $a_{1}+1 \equiv k(\bmod 2)$, Proposition 14 implies that $G$ contains a pivot-minor isomorphic to $C_{k}$.

\section{Proof of Theorem 6}

First we choose $\alpha>0$ and $\varepsilon_{0}>0$ so that

$$
4 \alpha \leqslant \alpha\left(\left\lceil\frac{3}{2} k+6\right\rceil\right) \text { and } \varepsilon_{0}=\varepsilon\left(\left\lceil\frac{3}{2} k+6\right\rceil\right) \text { where } \alpha(\cdot), \varepsilon(\cdot) \text { are specified in Lemma } 13
$$

and in addition $\alpha<1 /(8 k)$ as well. Let $\delta>0$ be a constant obtained by applying Corollary 10 with $\alpha / 3$ as $\alpha$ and $C_{k}$ as $H$. Choose $\varepsilon>0$ so that

$$
\varepsilon<\min \left(\frac{\delta}{12},(1-4(k+3) \alpha) \frac{\delta}{240}, \frac{\varepsilon_{0} \delta}{12}\right) .
$$

Let $n>1$ be an integer and $G$ be an $n$-vertex graph with no pivot-minor isomorphic to $C_{k}$. In particular, $G$ does not have $C_{k}$ as an induced subgraph. To derive a contradiction, we assume that $G$ contains no pure pair $(A, B)$ with $|A|,|B| \geqslant \varepsilon n$. We may assume that $\varepsilon n>1$, because otherwise an edge or a non-edge of $G$ gives a pure pair. 
By Corollary 10, there exists a subset $U$ of $V(G)$ such that $|U| \geqslant \delta|V(G)|$ and $\left.\Delta\left(G^{0}\right)[U]\right) \leqslant(\alpha / 3)|U|$ for some $G^{0} \in\{G, \bar{G}\}$. By the assumption on $G, G^{0}[U]$ has no pure pair $(A, B)$ with $|A|,|B| \geqslant(\varepsilon / \delta)|U|$. As $\varepsilon / \delta<1 / 3$, by Lemma $11, G^{0}[U]$ has a connected induced subgraph $G^{\prime}$ such that $\left|V\left(G^{\prime}\right)\right| \geqslant|U| / 3$. Let $n^{\prime}=\left|V\left(G^{\prime}\right)\right|$.

Then $n^{\prime} \geqslant(\delta / 3) n$ and $\Delta\left(G^{\prime}\right) \leqslant(\alpha / 3)|U| \leqslant \alpha n^{\prime}$. By the assumption on $G$,

$$
G^{\prime} \text { contains no pure pair }(A, B) \text { with }|A|,|B| \geqslant(3 \varepsilon / \delta) n^{\prime} .
$$

By applying Lemma 7 with $G^{\prime}$, we obtain a dominating induced tree $T$ and $r: V\left(G^{\prime}\right) \rightarrow$ $V(T)$ satisfying Lemma 7 (T1)-(T2) with $G^{\prime}$. For each $u \in V(T)$, let

$$
w(u):=\frac{\left|r^{-1}(\{u\})\right|}{n^{\prime}}
$$

be the weight of $u$. By applying Lemma 12 with the weight $w$, we obtain either an induced path $P$ of $T$ with weight at least $1 / 4$ or two unrelated sets $A$ and $B$ both with weight at least $1 / 4$.

In the latter case, Lemma 7 (T2) implies that $r^{-1}(A)$ is anticomplete to $r^{-1}(B)$ in $G^{\prime}$ and $\left|r^{-1}(A)\right|,\left|r^{-1}(B)\right| \geqslant n^{\prime} / 4 \geqslant(3 \varepsilon / \delta) n^{\prime}$, contradicting (2).

Hence, there exists an induced path $P$ in $G^{\prime}$ with $\left|V(P) \cup N_{G^{\prime}}(V(P))\right| \geqslant n^{\prime} / 4$. Let $W:=V(P) \cup N_{G^{\prime}}(V(P))$. Note that $n^{\prime} / 4 \geqslant \delta n / 12>\varepsilon n>1$ and so $|W| \geqslant 2$.

Suppose that $G^{\prime}$ is an induced subgraph of $\bar{G}$. Using (1), we apply Lemma 13 to $G^{\prime}[W]$ with $4 \alpha$ as $\alpha$ and $\left\lceil\frac{3}{2} k+6\right\rceil$ as $k$. Then we can deduce from (2) and $\varepsilon^{\prime}|W| \geqslant$ $\frac{12 \varepsilon}{\delta} \frac{n^{\prime}}{4}=(3 \varepsilon / \delta) n^{\prime}$ that the graph $G^{\prime}[W]$ contains an induced cycle $C_{m}$ with $m \geqslant\left\lceil\frac{3}{2} k+6\right\rceil$ and by Proposition $15, \overline{G^{\prime}}$ contains a pivot-minor isomorphic to $C_{k}$, and so does $G$, a contradiction.

Thus $G^{\prime}$ is an induced subgraph of $G$. Let $G^{*}:=G^{\prime}[W]$ and let $n^{*}=|W|$. Then $G^{*}$ has no pivot-minor isomorphic to $C_{k}, n^{*} \geqslant n^{\prime} / 4$, and $\Delta\left(G^{*}\right) \leqslant 4 \alpha n^{*}$. By (2), $G^{*}$ contains no pure pair $(A, B)$ with $|A|,|B| \geqslant(12 \varepsilon / \delta) n^{*}$. Now the theorem follows from applying the following lemma with $G^{*}, n^{*}, 4 \alpha, 12 \varepsilon / \delta$ playing the roles of $G, n, \alpha, \varepsilon$ respectively in the statement of the lemma.

Lemma 18. Let $k \geqslant 3$ be an integer. Let $0<\alpha<1 /(2 k), 0<\varepsilon \leqslant(1-(k+3) \alpha) / 20$. Let $G$ be a graph on $n \geqslant 2$ vertices such that $\Delta(G) \leqslant \alpha$ and $G$ has no pure pair $(A, B)$ with $|A|,|B| \geqslant \varepsilon n$. If $G$ has a dominating induced path $P$, then $G$ has a pivot-minor isomorphic to $C_{k}$.

Proof. Suppose that $G$ has no pivot-minor isomorphic to $C_{k}$. Note that $\varepsilon n>1$ as otherwise we have a pure pair on two vertices since $n \geqslant 2$. Let us label vertices of $P$ by 1,2 , $\ldots, s$ in the order.

As $P$ is a dominating path of $G$ and $1 \leqslant \Delta(G) \leqslant \alpha n$, we have $2 \alpha n s \geqslant(\alpha n+1) s \geqslant n$ and therefore

$$
s \geqslant 1 /(2 \alpha) \text {. }
$$

Note that $s-k>0$ because $\alpha<\frac{1}{2 k}$. As $P$ is an induced path, it contains a pure pair $(A, B)$ with $|A|,|B| \geqslant\left\lfloor\frac{s-1}{2}\right\rfloor$ and so $\frac{s-2}{2} \leqslant\left\lfloor\frac{s-1}{2}\right\rfloor<\varepsilon n$. Because $\varepsilon n>1$, we have 
$2 \varepsilon n+2<4 \varepsilon n$ and so

$$
s<2 \varepsilon n+2<4 \varepsilon n \text {. }
$$

Now, for each $i \in[s-k+1]$, let

$$
U_{i}^{-}:=\{1, \ldots, i-1\}, \quad U_{i}^{0}:=\{i, \ldots, i+k-1\}, \text { and } U_{i}^{+}:=\{i+k, \ldots, s\} .
$$

In other words, this partitions $P$ into three (possibly empty) subpaths. Furthermore, for all $i \in[s-k+1]$ and $u \in N_{G}\left(U_{i}^{-}\right)-V(P)$, let

$$
m_{i}^{-}(u):=\max \left(N_{G}(u) \cap U_{i}^{-}\right)
$$

and for all $i \in[s-k+1]$ and $u \in N_{G}\left(U_{i}^{+}\right)-V(P)$, let

$$
m_{i}^{+}(u):=\min \left(N_{G}(u) \cap U_{i}^{+}\right),
$$

indicating the largest neighbor of $u$ in $U_{i}^{-}$and the smallest neighbor of $u$ in $U_{i}^{+}$respectively. For each $i \in[s-k+1]$, let

$$
\begin{aligned}
& A_{i}:=N_{G}\left(U_{i}^{0}\right)-V(P) \text { and } \\
& B_{i}:=\left(N_{G}\left(U_{i}^{-}\right) \cap N_{G}\left(U_{i}^{+}\right)\right)-\left(A_{i} \cup V(P)\right) .
\end{aligned}
$$

Note that for each $u \in B_{i}$, we have

$$
m_{i}^{+}(u)-m_{i}^{-}(u) \not \equiv k \quad(\bmod 2)
$$

because otherwise $\left(u, m_{i}^{-}(u), m_{i}^{-}(u)+1, \ldots, m_{i}^{+}(u), u\right)$ forms an induced cycle of length at least $k$ and Proposition 14 implies that $G$ contains a pivot-minor isomorphic to $C_{k}$, a contradiction.

For each $i \in[s-k+1]$, let

$$
\begin{aligned}
& C_{i}^{1}:=\left\{u \in N_{G}\left(U_{i}^{-}\right)-\left(A_{i} \cup B_{i} \cup V(P)\right): m_{i}^{-}(u) \equiv 1 \quad(\bmod 2)\right\}, \\
& C_{i}^{2}:=\left\{u \in N_{G}\left(U_{i}^{-}\right)-\left(A_{i} \cup B_{i} \cup V(P)\right): m_{i}^{-}(u) \equiv 0 \quad(\bmod 2)\right\}, \\
& D_{i}^{1}:=\left\{u \in N_{G}\left(U_{i}^{+}\right)-\left(A_{i} \cup B_{i} \cup V(P)\right): m_{i}^{+}(u) \equiv k \quad(\bmod 2)\right\}, \text { and } \\
& D_{i}^{2}:=\left\{u \in N_{G}\left(U_{i}^{+}\right)-\left(A_{i} \cup B_{i} \cup V(P)\right): m_{i}^{+}(u) \equiv k+1 \quad(\bmod 2)\right\} .
\end{aligned}
$$

Recall that $P$ is dominating. Hence, for each $i$, the sets $\left\{A_{i}, B_{i}, C_{i}^{1}, C_{i}^{2}, D_{i}^{1}, D_{i}^{2}, V(P)\right\}$ forms a partition of $V(G)$ into 7 possibly empty sets.

If there exists an edge between $u \in C_{i}^{j}$ and $v \in D_{i}^{j}$ for some $j \in[2]$, then we obtain an induced cycle $\left(u, m_{i}^{-}(u), m_{i}^{-}(u)+1, \ldots, m_{i}^{+}(v), v, u\right)$ having length $m_{i}^{+}(v)-m_{i}^{-}(u)+3>k$ and $m_{i}^{+}(v)-m_{i}^{-}(u)+3 \equiv k(\bmod 2)$, contradicting our assumption that $G$ has no pivotminor isomorphic to $C_{k}$ by Proposition 14. Thus $C_{i}^{j}$ is anticomplete to $D_{i}^{j}$. Hence,

$$
\min \left\{\left|C_{i}^{j}\right|,\left|D_{i}^{j}\right|\right\}<\varepsilon n .
$$

for all $i \in[s-k+1]$ and $j \in[2]$. Furthermore, we prove the following. 


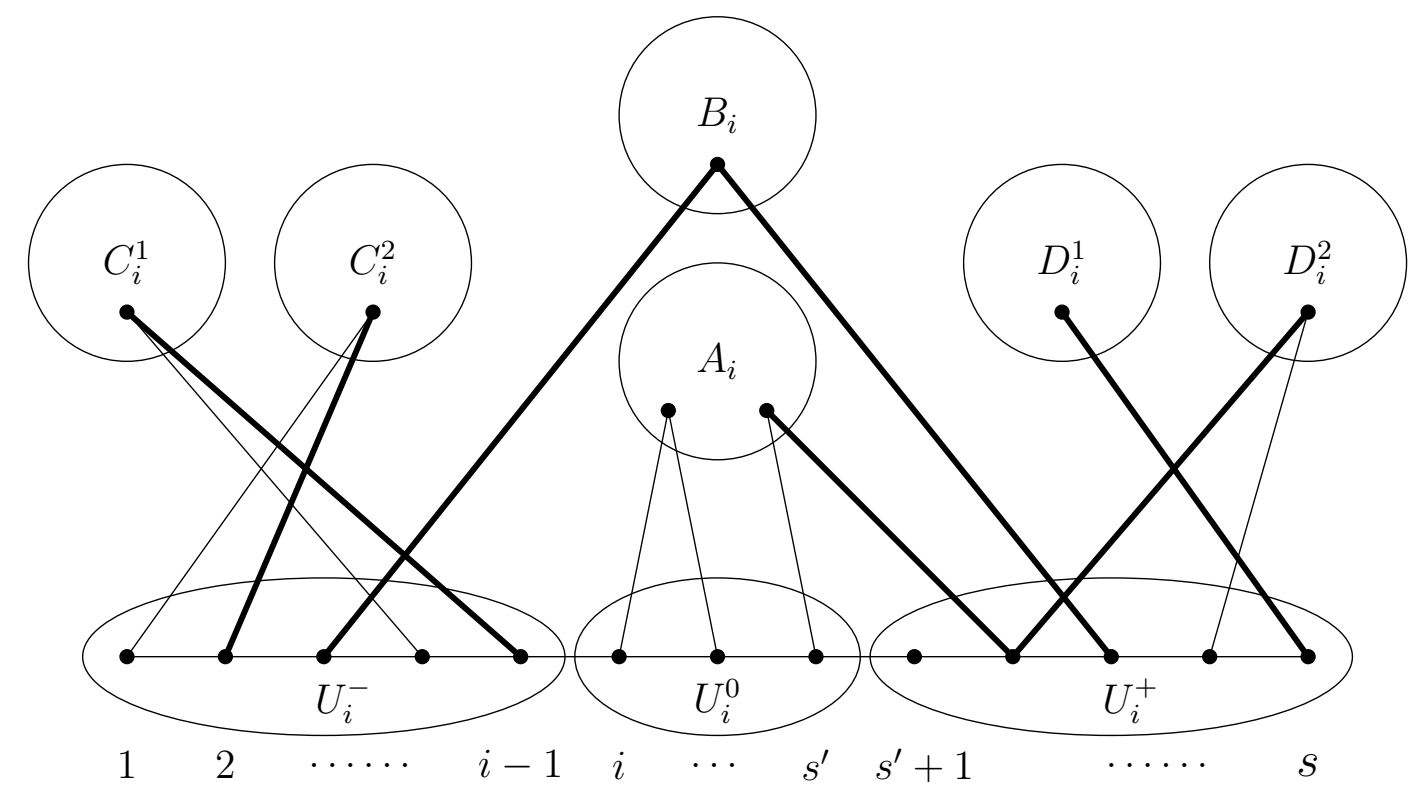

Figure 3: $s^{\prime}=i+k-1$. Bold lines indicate $m_{i}^{-}(u)$ and $m_{i}^{+}(u)$.

Claim 19. Let $i \in[s-k+1]$. For each $v \in B_{i}$, all integers in $N_{G}(v) \cap U_{i}^{-}$have the same parity and all integers in $N_{G}(v) \cap U_{i}^{+}$have the same parity.

Proof of Claim 19. If $N_{G}(v) \cap U_{i}^{+}$has two integers $a<b$ of the different parity, then $G$ contains a strongly $k$-good generalized fan by taking a subpath of $P$ from $m_{i}^{-}(v)$ to $b$ as its main path and $v$ as its center. Then by Proposition 17, $G$ contains a pivot-minor isomorphic to $C_{k}$, contradicting the assumption. Thus all integers in $N_{G}(v) \cap U_{i}^{+}$have the same parity and similarly all integers in $N_{G}(v) \cap U_{i}^{-}$have the same parity.

Claim 20. For all $i \in[s-k+1],\left|B_{i}\right|<2(\alpha+2 \varepsilon) n$.

Proof of Claim 20. Suppose $\left|B_{i}\right| \geqslant 2(\alpha+2 \varepsilon) n$ for some $i \in[s-k+1]$. Then there exists $r_{B} \in\{0,1\}$ such that

$$
B^{\prime}:=\left\{u \in B_{i}: m_{i}^{-}(u) \equiv r_{B} \quad(\bmod 2)\right\}
$$

has size at least $(\alpha+2 \varepsilon) n$. By $(4), m_{i}^{+}(u) \equiv k+r_{B}+1(\bmod 2)$ for all $u \in B^{\prime}$.

We claim that if $u v$ is an edge in $G\left[B^{\prime}\right]$, then $\left(m_{i}^{-}(u), m_{i}^{+}(u)\right)=\left(m_{i}^{-}(v), m_{i}^{+}(v)\right)$. Suppose not. Without loss of generality, we may assume that $m_{i}^{-}(u)<m_{i}^{-}(v)$, because otherwise we may reverse the ordering of $P$ to ensure that $m_{i}^{-}(u) \neq m^{-}(v)$ and swap $u$ and $v$ if necessary.

If $m_{i}^{+}(u) \geqslant m_{i}^{+}(v)$, then by Claim $19,\left\{m_{i}^{-}(v), m_{i}^{-}(v)+1, \ldots, m_{i}^{+}(u), u, v\right\}$ induces a strongly $k$-good generalized fan with $v$ as a center and $\left(m_{i}^{-}(v), m_{i}^{-}(v)+1, \ldots, m_{i}^{+}(u), u\right)$ as its main path. This implies that $G$ has a pivot-minor isomorphic to $C_{k}$ by Proposition 17 , contradicting our assumption. 
If $m_{i}^{+}(u)<m_{i}^{+}(v)$, then $\left(m_{i}^{-}(v), m_{i}^{-}(v)+1, \ldots, m_{i}^{+}(u), u, v\right)$ is an induced cycle of length $m_{i}^{+}(u)-m_{i}^{-}(v)+3 \geqslant k$, and $m_{i}^{+}(u)-m_{i}^{-}(v)+3 \equiv\left(k+r_{B}+1\right)-r_{B}+3 \equiv k$ $(\bmod 2)$, a contradiction by Proposition 14.

Hence, $\left(m_{i}^{-}(u), m_{i}^{+}(u)\right)=\left(m_{i}^{-}(v), m_{i}^{+}(v)\right)$ for all $u v \in E\left(G\left[B^{\prime}\right]\right)$. Let $C_{1}, \ldots, C_{t}$ be the connected components of $G\left[B^{\prime}\right]$. By the above observation, for each $j \in[t]$, there exist $a_{j} \in U_{i}^{-}$and $b_{j} \in U_{i}^{+}$such that $V\left(C_{j}\right) \subseteq N_{G}\left(a_{j}\right) \cap N_{G}\left(b_{j}\right)$. So, $\left|V\left(C_{j}\right)\right| \leqslant \alpha n$. As $\left|B^{\prime}\right| \geqslant(\alpha+2 \varepsilon) n$, there exists a set $I \subseteq\{1,2, \ldots, t\}$ such that $\varepsilon n \leqslant\left|\bigcup_{i \in I} V\left(C_{i}\right)\right| \leqslant(\alpha+\varepsilon) n$. Let $A:=\bigcup_{i \in I} V\left(C_{i}\right)$ and $B:=B^{\prime}-A$. Then $(A, B)$ is a pure pair of $G$ with $|A|,|B| \geqslant \varepsilon n$, a contradiction.

Claim 21. There exist $i_{*} \in[s-k+1]$ and $j_{*} \in[2]$ such that

$$
\left|C_{i_{*}}^{j_{*}}\right|,\left|D_{i_{*}}^{3-j_{*}}\right| \geqslant 3 \varepsilon n
$$

Proof of Claim 21. First, since $\Delta(G) \leqslant \alpha n,\left|A_{i}\right| \leqslant k \alpha n$ for each $i \in[s-k+1]$.

Let $f(i):=\left|C_{i}^{1}\right|+\left|C_{i}^{2}\right|$. Then

$$
\begin{aligned}
f(1) & =0 \\
f(s-k+1) & =n-\left|A_{s-k+1}\right|-s \quad \text { because } U_{s-k+1}^{+}=D_{s-k+1}^{1}=D_{s-k+1}^{2}=B_{s-k+1}=\varnothing, \\
& \geqslant n-k \alpha n-4 \varepsilon n \quad \text { by }(3) \text { and the assumption that } \Delta(G) \leqslant \alpha n, \\
& =(1-k \alpha-4 \varepsilon) n \geqslant 6 \varepsilon n,
\end{aligned}
$$

and for each $i \in[s-k]$, we have

$$
f(i+1)-f(i) \leqslant \operatorname{deg}_{G}(i) \leqslant \alpha n .
$$

Hence, there exists $i_{*} \in[s-k+1]$ such that $6 \varepsilon n \leqslant f\left(i_{*}\right)<(6 \varepsilon+\alpha) n$. As $\left|B_{i_{*}}\right|<2(\alpha+2 \varepsilon) n$, we have

$$
\begin{aligned}
\left|D_{i_{*}}^{1}\right|+\left|D_{i_{*}}^{2}\right| & =n-\left|A_{i_{*}}\right|-\left|B_{i_{*}}\right|-\left(\left|C_{i_{*}}^{1}\right|+\left|C_{i_{*}}^{2}\right|\right)-|V(P)| \\
& \geqslant n-k \alpha n-2(\alpha+2 \varepsilon) n-(6 \varepsilon+\alpha) n-4 \varepsilon n \\
& =(1-(k+3) \alpha-14 \varepsilon) n \geqslant 6 \varepsilon n .
\end{aligned}
$$

So, there exist $a, b \in\{1,2\}$ such that $\left|C_{i_{*}}^{a}\right|,\left|D_{i_{*}}^{b}\right| \geqslant 3 \varepsilon n$. By (5), $a \neq b$ and so we take $j_{*}:=a$. This proves the claim.

Claim 22. For each component $C$ of $G\left[C_{i_{*}}^{j_{*}}\right]$ and each component $D$ of $G\left[D_{i_{*}}^{3-j_{*}}\right],(C, D)$ is a pure pair of $G$.

Proof of Claim 22. Assume not. By symmetry, we may assume that $C$ has a vertex $u$ having both a neighbor and a non-neighbor in $D$, because otherwise we swap $C$ and $D$ by reversing the order of $P$. As $D$ is connected, there exist $v, v^{\prime} \in V(D)$ such that $u v, v v^{\prime} \in E(G)$ and $u v^{\prime} \notin E(G)$. 
Note that $m_{i_{*}}^{+}(v) \equiv m_{i_{*}}^{+}\left(v^{\prime}\right)(\bmod 2)$ and

$$
\text { for every neighbor } \ell \in N_{G}(v) \cap U_{i_{*}}^{+} \text {, the number } \ell-m_{i_{*}}^{+}(v) \text { is even, }
$$

because otherwise for the minimum $\ell \in N_{G}(v) \cap U_{i^{*}}^{+}$with odd $\ell-m_{i_{*}}^{+}(v)$, a vertex set $\left\{v, m_{i_{*}}^{-}(u), m_{i_{*}}^{-}(u)+1, \ldots, \ell, u\right\}$ induces a strongly $k$-good generalized fan with $v$ as its center, a contradiction by Proposition 17.

If $m_{i_{*}}^{+}(v) \leqslant m_{i_{*}}^{+}\left(v^{\prime}\right)$, then $\left\{v, u, m_{i_{*}}^{-}(u), m_{i_{*}}^{-}(u)+1, \ldots, m_{i_{*}}^{+}\left(v^{\prime}\right), v^{\prime}\right\}$ induces a strongly $k$-good generalized fan with $v$ as a center by $(6)$.

If $m_{i_{*}}^{+}(v)>m_{i_{*}}^{+}\left(v^{\prime}\right)$, then simply $\left(u, m_{i_{*}}^{-}(u), m_{i_{*}}^{-}(u)+1, \ldots, m_{i_{*}}^{+}\left(v^{\prime}\right), v^{\prime}, v, u\right)$ is an induced cycle whose length is at least $k$ and is of the same parity with $k$. Hence Proposition 14 implies a contradiction.

By Claim 22, there exists $S \in\left\{C_{i_{*}}^{j_{*}}, D_{i_{*}}^{3-j_{*}}\right\}$ such that every component of $G[S]$ has less than $\varepsilon n$ vertices. By Claim 21, we can greedily find a set of components of $G[S]$ covering at least $\varepsilon n$ vertices and at most $2 \varepsilon n$ vertices. Since $|S| \geqslant 3 \varepsilon n$, the vertices of $S$ covered by this set of components with the vertices of $S$ not covered by this set of components give a pure pair $(A, B)$ with $|A|,|B| \geqslant \varepsilon n$, a contradiction. This proves the lemma.

\section{Discussions}

For a graph $G$, we write $\chi(G)$ to denote its chromatic number and $\omega(G)$ to denote its clique number, that is the maximum size of a clique. A class $\mathcal{G}$ of graphs is called $\chi$ bounded if there exists a function $f: \mathbb{Z} \rightarrow \mathbb{Z}$ such that for every induced subgraph $H$ of a graph in $\mathcal{G}, \chi(H) \leqslant f(\omega(H))$. In addition, we say $\mathcal{G}$ is polynomially $\chi$-bounded if $f$ can be taken as a polynomial.

Every polynomially $\chi$-bounded class of graphs has the strong Erdös-Hajnal property, but the converse does not hold; see the survey paper by Scott and Seymour [22]. So it is natural to ask whether the class of graphs with no pivot-minor isomorphic to $C_{k}$ is polynomially $\chi$-bounded, which is still open. So far Choi, Kwon, and Oum [4] showed that it is $\chi$-bounded.

Theorem 23 (Choi, Kwon, and Oum [4, Theorem 4.1]). For each $k \geqslant 3$, the class of graphs with no pivot-minor isomorphic to $C_{k}$ is $\chi$-bounded.

They showed that $\chi(G) \leqslant 2\left(6 k^{3}-26 k^{2}+25 k-1\right)^{\omega(G)-1}$ holds for graphs $G$ having no pivot-minor isomorphic to $C_{k}$, far from being a polynomial. Theorem 23 is now implied by a recent theorem of Scott and Seymour [21], solving three conjectures of Gyárfás [13] on $\chi$-boundedness all at once.

Theorem 24 (Scott and Seymour [21]). For all $k \geqslant 0$ and $\ell>0$, the class of all graphs having no induced cycle of length $k$ modulo $\ell$ is $\chi$-bounded.

To see why Theorem 24 implies Theorem 23, take $\ell:=2\lceil k / 2\rceil$ and apply Proposition 14. Still the bound obtained from Theorem 24 is far from being a polynomial.

And yet no one was able to answer the following problem of Esperet. 
Problem 25 (Esperet; see [15]). Is it true that every $\chi$-bounded class of graphs polynomially $\chi$-bounded?

Thus it is natural to pose the following conjecture.

Conjecture 26. For every graph $H$, the class of graphs with no pivot-minor isomorphic to $H$ is polynomially $\chi$-bounded.

It is open whether Conjecture 26 holds when $H=C_{k}$. Conjecture 26 implies not only Conjectures 3, 5 but also the following conjecture of Geelen (see [8]) proposed in 2009 at the DIMACS workshop on graph colouring and structure held at Princeton University.

Conjecture 27 (Geelen; see [8]). For every graph $H$, the class of graphs with no vertexminor isomorphic to $H$ is $\chi$-bounded.

Of course it is natural to pose the following conjecture, weaker than Conjecture 26 but stronger than Conjecture 27.

Conjecture 28 (Kim, Kwon, Oum, and Sivaraman [16]). For every graph $H$, the class of graphs with no vertex-minor isomorphic to $H$ is polynomially $\chi$-bounded.

For vertex-minors, more results are known. Kim, Kwon, Oum, and Sivaraman [16] proved that for each $k \geqslant 3$, the class of graphs with no vertex-minor isomorphic to $C_{k}$ is polynomially $\chi$-bounded. Their theorem is now implied by the following two recent theorems. To describe these theorems, we first have to introduce a few terms. A circle graph is the intersection graph of chords in a circle. In particular, $C_{k}$ is a circle graph. The rank-width of a graph is one of the width parameters of graphs, measuring how easy it is to decompose a graph into a tree-like structure while keeping every cut to have a small 'rank'. Rank-width was introduced by Oum and Seymour [19]. We will omit the definition of the rank-width.

Theorem 29 (Geelen, Kwon, McCarty, and Wollan [12]). For each circle graph $H$, there is an integer $r(H)$ such that every graph with no vertex-minor isomorphic to $H$ has rankwidth at most $r(H)$.

Theorem 30 (Bonamy and Pilipczuk [3]). For each $k$, the class of graphs of rank-width at most $k$ is polynomially $\chi$-bounded.

As noted in [6], it is easy to prove directly that the class of graphs of bounded rankwidth has the strong Erdős-Hajnal property, without using Theorem 30. See Figure 4 for a diagram showing the containment relations between these properties.

So, one may wonder whether the class of graphs with no pivot-minor isomorphic to $C_{k}$ has bounded rank-width. Unfortunately, if $k$ is odd, then it is not true, because all bipartite graphs have no pivot-minor isomorphic to $C_{k}$ for odd $k$ and yet have unbounded rank-width, see [17]. If $k$ is even, then it would be true if the following conjecture hold.

Conjecture 31 (Oum [18]). For every bipartite circle graph $H$, there is an integer $r(H)$ such that every graph with no pivot-minor isomorphic to $H$ has rank-width at most $r(H)$. 


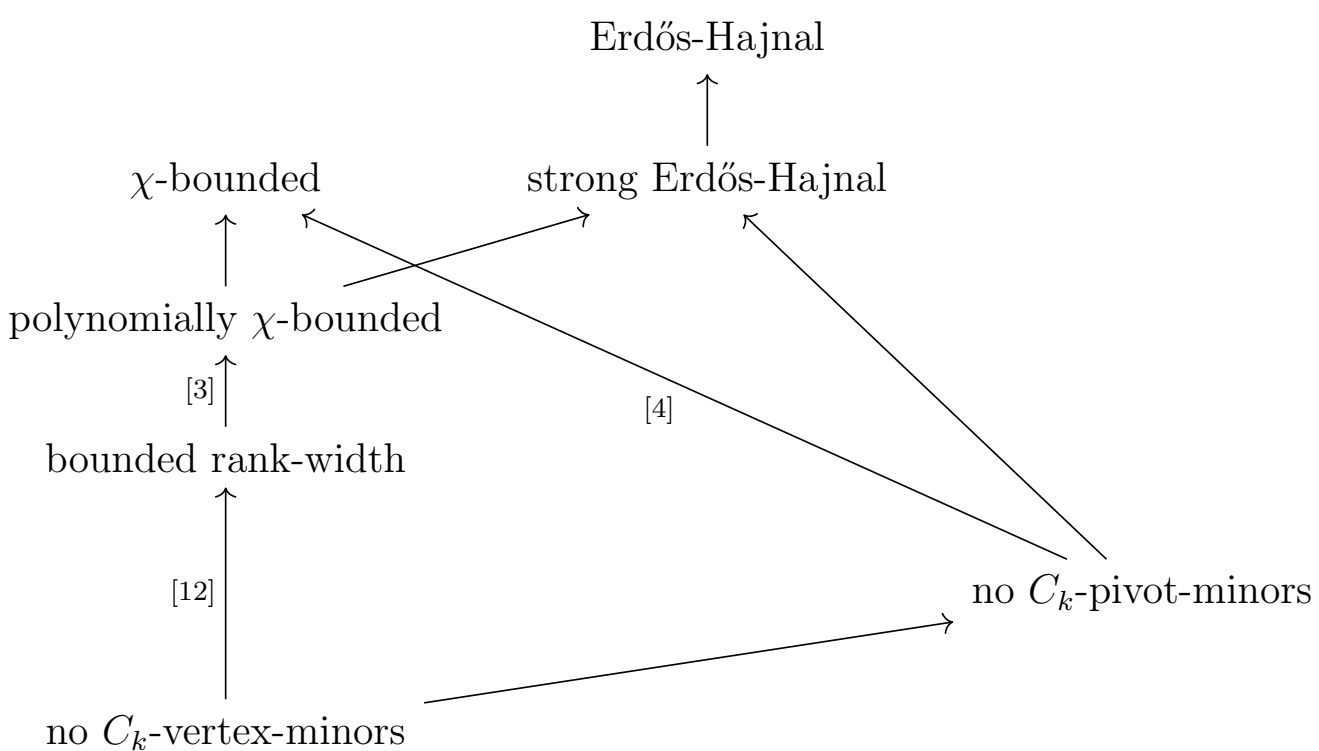

Figure 4: Known implications between properties of classes of graphs.

\section{Note.}

Chudnovsky, Scott, Seymour, and Spirkl [7] proved that for every graph $H$, the class of graphs $G$ such that neither $G$ nor $\bar{G}$ has any subdivision of $H$ as an induced subgraph has the strong Erdős-Hajnal property. This implies that when $k$ is even, the class of graphs with no induced even hole of length at least $k$ and no induced even anti-hole of length at least $k$ has the strong Erdös-Hajnal property. This is because every subdivision of a large theta graph ${ }^{2}$ contains a large even hole. This implies Theorem 4 for even $k$ but not for odd $k$ by Propositions 14 and 15. The authors would like to thank the authors of [7] to share this observation.

\section{Acknowledgement}

The authors would like to thank anonymous reviewers for their careful reading and helpful suggestions.

\section{References}

[1] N. Alon, J. Pach, R. Pinchasi, R. Radoičić, and M. Sharir. Crossing patterns of semi-algebraic sets. J. Combin. Theory Ser. A, 111(2):310-326, 2005. https://doi.org/10.1016/j.jcta.2004.12.008.

\footnotetext{
${ }^{2}$ A theta graph is a graph consisting of three internally disjoint paths of length at least 1 joining two fixed vertices.
} 
[2] M. Bonamy, N. Bousquet, and S. Thomassé. The Erdős-Hajnal conjecture for long holes and antiholes. SIAM J. Discrete Math., 30(2):1159-1164, 2016. https://doi.org/10.1137/140981745.

[3] M. Bonamy and M. Pilipczuk. Graphs of bounded cliquewidth are polynomially $\chi$-bounded. Adv. Comb., Paper No. 8, 21 pp., 2020. https://doi.org/10.19086/aic.13668.

[4] I. Choi, O. Kwon, and S. Oum. Coloring graphs without fan vertex-minors and graphs without cycle pivot-minors. J. Combin. Theory Ser. B, 123:126-147, 2017. https://doi.org/10.1016/j.jctb.2016.11.007.

[5] M. Chudnovsky. The Erdös-Hajnal conjecture -a survey. J. Graph Theory, 75(2):178-190, 2014. https://doi.org/10.1002/jgt.21730.

[6] M. Chudnovsky and S. Oum. Vertex-minors and the Erdős-Hajnal conjecture. Discrete Math., 341(12):3498-3499, 2018. https://doi.org/10.1016/j.disc.2018.09.007.

[7] M. Chudnovsky, A. Scott, P. Seymour, and S. Spirkl. Pure pairs. II. Excluding all subdivisions of a graph. arXiv:1804.01060, 2020.

[8] Z. Dvořák and D. Král. Classes of graphs with small rank decompositions are $\chi$ bounded. European J. Combin., 33(4):679-683, 2012. https://doi.org/10.1016/j.ejc.2011.12.005.

[9] P. Erdős and A. Hajnal. Ramsey-type theorems. Discrete Appl. Math., 25(1-2):37-52, 1989. Combinatorics and complexity (Chicago, IL, 1987).

[10] J. Fox and J. Pach. Erdős-Hajnal-type results on intersection patterns of geometric objects. In Horizons of combinatorics, volume 17 of Bolyai Soc. Math. Stud., pages 79-103. Springer, Berlin, 2008. https://doi.org/10.1007/978-3-540-77200-2_4.

[11] J. Fox and B. Sudakov. Induced Ramsey-type theorems. Adv. Math., 219(6):17711800, 2008. https://doi.org/10.1016/j.aim.2008.07.009.

[12] J. Geelen, O. Kwon, R. McCarty, and P. Wollan. The grid theorem for vertex-minors. J. Combin. Theory Ser. B, 2020. https://doi.org/10.1016/j .jctb.2020.08.004.

[13] A. Gyárfás. Problems from the world surrounding perfect graphs. In Proceedings of the International Conference on Combinatorial Analysis and its Applications (Pokrzywna, 1985), volume 19, pages 413-441 (1988), 1987.

[14] M. Kamiński, V. V. Lozin, and M. Milanič. Recent developments on graphs of bounded clique-width. Discrete Appl. Math., 157(12):2747-2761, 2009. https://doi.org/10.1016/j.dam.2008.08.022.

[15] T. Karthick and F. Maffray. Vizing bound for the chromatic number on some graph classes. Graphs Combin., 32(4):1447-1460, 2016. https://doi.org/10.1007/s00373-015-1651-1.

[16] R. Kim, O. Kwon, S. Oum, and V. Sivaraman. Classes of graphs with no long cycle as a vertex-minor are polynomially $\chi$-bounded. J. Combin. Theory Ser. B, 140:372-386, 2020. https://doi.org/10.1016/j.jctb.2019.06.001. 
[17] S. Oum. Rank-width and vertex-minors. J. Combin. Theory Ser. B, 95(1):79-100, 2005. https://doi.org/10.1016/j.jctb.2005.03.003.

[18] S. Oum. Excluding a bipartite circle graph from line graphs. J. Graph Theory, 60(3):183-203, 2009. https://doi.org/10.1002/jgt. 20353.

[19] S. Oum and P. Seymour. Approximating clique-width and branch-width. J. Combin. Theory Ser. B, 96(4):514-528, 2006. https://doi.org/10.1016/j.jctb.2005.10.006.

[20] V. Rödl. On universality of graphs with uniformly distributed edges. Discrete Math., 59(1-2):125-134, 1986. https://doi.org/10.1016/0012-365X (86) 90076-2.

[21] A. Scott and P. Seymour. Induced subgraphs of graphs with large chromatic number. X. Holes of specific residue. Combinatorica, 39(5):1105-1132, 2019. https://doi.org/10.1007/s00493-019-3804-y.

[22] A. Scott and P. Seymour. A survey of $\chi$-boundedness. J. Graph Theory, 95(3):473504, 2020. https://doi.org/10.1002/jgt. 22601. 